\title{
ESTROMATOLITOS "GYMNOSOLENIDOS" EN EL LADO ORIENTAL DE MINAS GERAIS, BRASIL
}

\author{
HUMBERTO GERVASIO MARCHESE*
}

\begin{abstract}
The described outcrop is the first discovered in the eastern part of Minas Gerais State. In the present work, after an analysis of the different classification criteria, the described stromatolites are classified on the basis of environmental and/or chronological criteria and considered to belong to the Gymnosolenida supergroup. of Upper Ripean age (950 to 650 m.y.). The present data, together with previous paleontological works permit us to place the Bambui Group within the 1000 to 650 m.y. time span (from the upper part of the Medium Riphean to the Upper Riphean).
\end{abstract}

RESUMO O jazigo de estromatólitos descrito é o primeiro descoberto na região oriental de Minas Gerais. No presente trabalho, após ser feita a análise dos diversos critérios de classificação, os estromatólitos descritos são classificados com base em critérios ambientais e/ou cronológicos e atribuídos ao supergrupo Gymnosolenida, do Rifeano Superior (950 a 650 m.a.). Com base nos resultados do presente trabalho e de achados paleontológicos anteriores, atribui-se ao Grupo Bambuí idade compreendida no intervalo de 1000 a 650 m.a., ou seja, entre a parte alta do Rifeano Médio e o Rifeano Superior.

INTRODUCCION Este estudio forma parte de uno mayor realizado en informe separado (Marchese, 1973) y en el cual se ha desarrollado más extensamente las características petrológicas ambientales de las sedimentitas del Grupo Bambuí. Además de dar a conocer un nuevo yacimiento estromatolítico, en este estudio se sintetizan todos los hallazgos paleontológicos del Grupo Bambuí, se discute la validez de su utilización, se esbozan y utilizan las principales clasificaciones en uso de las diferentes escuelas y se analizan los elementos de juicio para asignar edad a las sedimentitas. Como antecedentes de hallazgos paleontológicos anteriores, debemos citar el area occidental (definida como de mayor inestabilidad tectosedimentaria y tectónica - Marchese, 1973), los estudios de Cassedane (1968), Dardenne y otros (1970), Dardenne (1972), Cloud y Dardenne (1973).

En la denominada area oriental (mayor estabilidad tectosedimentaria) el yacimiento descripto en este trabajo constituye la primera mención de estructuras estromatolíticas en el lado oriental de Minas Gerais. Otros datos paleontológicos de la misma área son los hallazgos de Sommer (1969, 1971) constituidos por microfósiles y bioglifos, y de Casedanne (1965) con el encuentro de texturas algales. Este mismo autor (1964) halló en el estado de Bahia, "Collenias" dentro de las sedimentitas del Grupo Bambuí. Todas estas referencias y sus conclusiones principales se encuentran resumidas en el Cuadro II.

El significado del término estromatolito ha sido discutido por diferentes autores, y alcanza más de quince definiciones, algunas de las cuales podemos encontrar en el excelente trabajo de recopilación y clasificación de Hofman (1969).

Tratando de sintetizar serían según lo expresado por los diferentes autores: "Estructuras organo-sedimentarias de variados tamaños (mm. a mts.) y normas (estratiforme, nodular

*Departamento de Ciencias Geológicas. Facultad de Ciencias Exactas y Naturales de la Universidad de Buenos Aires. Miembro de la Carrera del Investigador Científico del Consejo Nacional de Investigaciones Científicas y Técnicas. República Argentina 
o columnar), generalmente con texturas internas (láminas regulares e irregulares). Estas estructuras-texturas son la consecuencia de la interacción compleja de la actividad biológica de algas y/o microorganismos con el medio ambiente: composición, energía, material en suspensión, temperatura, etc."

La utilización de estas estructuras-texturas para la subdivisión estratigráfica del preCámbrico es muy controvertida. Por un lado se encuentran los que aconsejan su utilización únicamente como indicadores ambientales por considerar a estas estructuras orgánicas exclusivamente condicionadas por el medio ambiente. Dependiendo del mismo las formas van a variar sin importar las características específicas de la comunidad: es decir que diferentes asociaciones se comportarían igual y tendrían iguales formas en un mismo ambiente ecológico, y por el contrario, iguales comunidades se desarrollarían con diferentes formas en ambientes distintos. En el año 1964, Logan, Rezak y Ginsburg propusieron una clasificación basada en formas geométricas y su relación con el ambiente de crecimiento. Reconocen tres formas principales de estructuras con estromatolitos y oncolitos actuales: A) LLH, hemisferoides lateralmente enlazados (laterally linked hemispheroids); B) SSH, hemisferoides verticalmente apilados (discretes vertically hemispheroids stacked); C) SS, esferoides aislados, esferoides concéntricamente ordenados o hemisferoides apilados desordenadamente (discrete spheroids - either as ramdomly stacked hemispheroids or concentrically arranged spheroids).

Esta clasificación tiene diferentes variaciones y combinaciones entre las distintas fórmulas geométricas, y usando algunas de ellas en el denominador se puede caracterizar la estructura interna.

Otros estudios que también señalan la influencia ambiental sobre el crecimiento de los estromatolitos son los de Cheng, Chi-chiang y Kuo-chang (1969), Hubbard (1972), etc.

Por otra parte, se encuentran los autores, Cloud y Semikhatov (1969), Raaben (1969), Walter y Preiss (1972) y otros, principalmente rusos y australianos, que reconocen como válida la utilización de estas estructuras para la datación y zonación del preCámbrico.

Los criterios sustentados por estos autores son muy varios, entre los que merecen destacarse: Raaben (1969) y Cloud y Semikhatov (1969). Para el primero, su utilización en el preCámbrico se debe a: 1) la estabilidad de la secuencia de cambios en la asociación de estromatolitos columnares de diferentes regiones de U.S.S.R. y 2) la similitud de los caracteres en serie que tienen igual rango de intervalos de edad absoluta. Además este autor también sostiene que la utilización de estromatolitos columnares es preferible a los estratiformes por tener características mas discernibles en su estructura. También insiste en que las texturas de los microestratos es muy importante.

La clasificación realizada por Raaben (op. cit.) se basa en los siguientes caracteres morfológicos: a) formas de las columnas individuales (reconoce tres tipos principales), b) tamaño (diámetro de las columnas) aconseja el estudio bioestadístico para establecer intervalos de variaciones, puesto que la gran heterogeneidad en esta medida es muy amplia, inclusive en el mismo estromatolito, c) ramificaciones (no ramificados, ramificados activos y pasivos) y d) forma y textura del microestrato interno. Las características a y b parecen ser interdependientes. En base a las características morfológicas este autor divide a los estromatolitos columnares en cuatro supergrupos principales: 1) Conophytonida (no ramificado, con columnas cilíndricas), 2) Kussiellida (ramificación pasiva, columnas cilíndricas), 3) Tungussida (ramificación activa, columnas engrosándose hacia arriba en forma de taza (cup-like) y con ejes divergentes), 4) Gymnosolenida (ramificación activa, columnas pseudocilíndricas, ejes aproximadamente paralelos).

Según Cloud y Semikhatov (1969), los estromatolitos son conocidos en la columna geológica desde la actualidad hasta el preCámbrico (Prerifeano 2700 m.a.), la composición 
es principalmente calcárea o dolomítica, pero se encuentran algunos silíceos debido al efecto de reemplazo en diferentes etapas de diagénesis. Se presentan abundantemente y con gran variación de formas en el registro estatigráfico en rocas más antiguas que el Ordovícico temprano 480 m.a. y principalmente muy desarrollados en rocas prepaleozoicas jóvenes (1 650 a 650 m.a.).

Esta aparición los hace importantes para ser utilizados en zonaciones, debido a la ausencia de otros elementos fosilíferos convencionales. Aunque se conocen varias formas de estromatolitos que muestran las mismas variaciones a través de grandes períodos geológicos, es necesario adoptar precauciones en las interpretaciones de la morfología particular de cualquier tipo individual de estromatolitos que tengan implicancia geocronológica. La validez de la extrapolación de correlaciones mundiales, usando estromatolitos, esta siendo investigada, y en los últimos tiempos, la similitud sugerida por trabajos efectuados en E.E.U.U. de Norte América, Canadá, Africa y Australia permite esperar que éstos últimos puedan ser utilizados sin dudas para las correlaciones intercontinentales. Existen mas de 30 grupos con 100 formas descriptas.

Las clasificaciones de los estromatolitos propuestas por Cloud y Semikhatov (1969) se basa en la utilización de los estromatolitos en si mismos e independientemente de los organismos responsables de su formación (variación de asociación algas-bacterias o en la predominancia de unas sobre otras o de algunas comunidades monoespecíficas). Por lo tanto tomando como punto de partida la premisa anteriormente mencionada, la subdivisión de los grupos ("géneros") se basa en la siguiente combinación de caracteres: forma de las columnas, tipo de ramificación o cambio en el tipo de columnas (vertical o lateralmente), la morfología de la zona marginal y las características de la laminación interna (texturas). Esto último puede servir para diferenciar formas ("especies"). Estos autores analizan 12 grupos de estromatolitos columnares que son conocidos (o probables) en dos o mas continentes, tres de los cuales son descriptos por primera vez Eucasiphora, Gruneria y Katernia.

Esta controversia sobre la implicancia exclusivamente paleoecológica o cronoestratigráfica se ve incrementada por la dificultad del estudio de los estromatolitos, para la colección de muestras (grandes columnas) y/o variaciones muy continuas. Además, no siempre pueden hacerse buenas observaciones debido a la alteración superficial y diferencial de la roca que lo contiene, o a que la misma no tiene buenas exposiciones.

El estudio de campo debe basarse en mediciones estadísticas de las ramificaciones, tamaños, diámetros, formas de columnas y su relación con la roca que los alberga. Así, siguiendo las normas de clasificación de Cloud y Semikhatov (op. cit.) y Raaben (op. cit.), los grupos (géneros) o Supergrupos pueden ser determinados en el campo. Para llegar a un resultado que contemple especies, son necesarios los trabajos de laboratorio (analizar morfología exterior por secciones de perfiles seriados, ver Figs. 4 y 5 de este estudio), determinación de los tamaños y las características de las texturas, componentes de la laminación).

Hoffman (1969) propone una clasificación descriptiva sistematizada, que también usaremos para este trabajo, por considerarla de gran importancia en la unificación de criterios para la descripción de estromatolitos y su posterior clasificación.

Ubicacion geografica Los dos yacimientos encontrados y descriptos en este trabajo se hallan en las márgenes de la ruta BR-040 que une Belo Horizonte con Brasília, a 63 km de la salida de Belo Horizonte. El primero, ubicado mas al sur, se encuentra en la margen izquierda y en frente del entroncamiento de la Ruta BR-040 con el camino que viene de la localidad de Sete Lagoas (Fig. 1). El segundo se encuentra pasando el puesto policial 


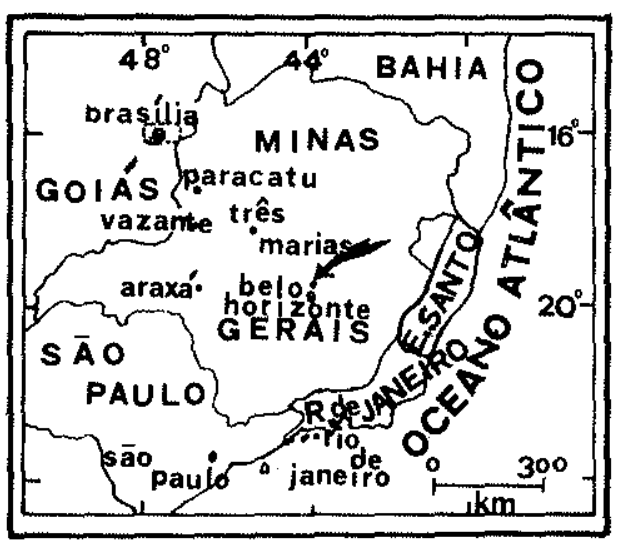

Figura 1 - Mapa de ubicación

(margen derecha) aproximadamente a 450 metros del primero y se presenta en ambas márgenes del camino, pero con mejor exposición del lado izquierdo (Foto 1).

Adoptaremos para el area de estudio la siguiente división del Grupo Bambuí de arriba hacia abajo:

Formación Três Marias (areniscas y limolitas)

Formación Santa Helena (limolita-arcillitas, bancos calcareos, pelitas calcareas)

Formación Sete Lagoas (calizas)

Este criterio está basado en que estas tres unidades tienen características definidas de nuestra area, lo cual no implica negar las variaciones laterales y verticales que se presenten en diferentes lugares de la cuenca. Ambos yacimientos estomatolíticos pertenecen aproximadamente a un mismo nivel estratigráfico, aunque se encuentran aislados subyaciendo (?) a las pelitas de la Formación Santa Helena. La diferencia más notable entre ambos afloramientos es que el meridional se encuentra mas afectado por fenómenos relacionados con dislocamientos locales (zona de falla?) y con numerosas intrusiones (venas) de sílice, carbonatos y brechamiento, que afectan a los estromatolitos y a las pelitas de la Formación Santa Helena. Estos procesos ya han sido descriptos por Branco y Costa (1961), en el $\mathrm{km} 82,5$ de su Roteiro y que coincide con la ubicación geográfica de este yacimiento. Es de hacer notar que la masa calcárea conteniendo las estructuras estromatolíticas parecen estar incluidas (?), dentro de la Formación Santa Helena.

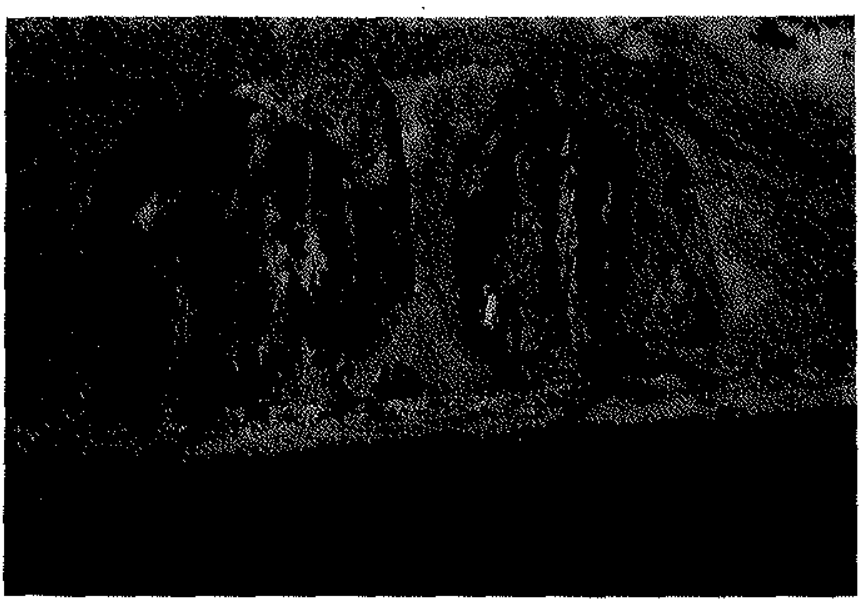

Foto 1 - Vista general del Yacimiento Norte 
Características macroscópicas Las dimensiones del yacimiento son:

Sur-aproximadamente 25 metros de ancho por 4 a 5 de alto

Norte-aproximadamente 20 metros de ancho por 5 a 6 de alto

El aspecto aislado de estos dos yacimientos, dentro (?) de las pelitas de la Formación Santa Helena, presenta características de estructuras biohermales-biostromales, pero un estudio estructural detallado puede confirmar si su relación es puramente tectónica. La disposición de los estromatolitos se presenta generalmente normal con una leve inclinación hacia el este (Fotos 2, 3, 4 y 5).

Las pelitas de la Formación Santa Helena tienen colores amarillos-verdosos, manchados superficialmente por material ferruginoso, que le da una tonalidad general rosada. Tiene laminación muy fina, de 1 a $3 \mathrm{~mm}$, con diaclasamiento y en parte con desarrollo de un plano S2. En algunas zonas las pelitas se encuentran "caidas" dentro de grietas de disolución de las calizas (Foto 1).

Las calizas son de color negro $\left(\mathrm{Nl}^{*}\right)$, en corte fresco se nota un leve cambio de tonalidades negro grisaceo $(\mathrm{N} 2 *)$, a gris oscuro $\left(\mathrm{N} 3{ }^{*}\right)$. La alteración diferencial y superficial de las calizas en algunas partes del yacimiento facilitó la observación y su estudio.

Las características petrográficas de los afloramientos marcan dos zonas: una esparítica, inequigranular-equigranular-hipidiotópica, subeuhedral, manchada por material ferru-

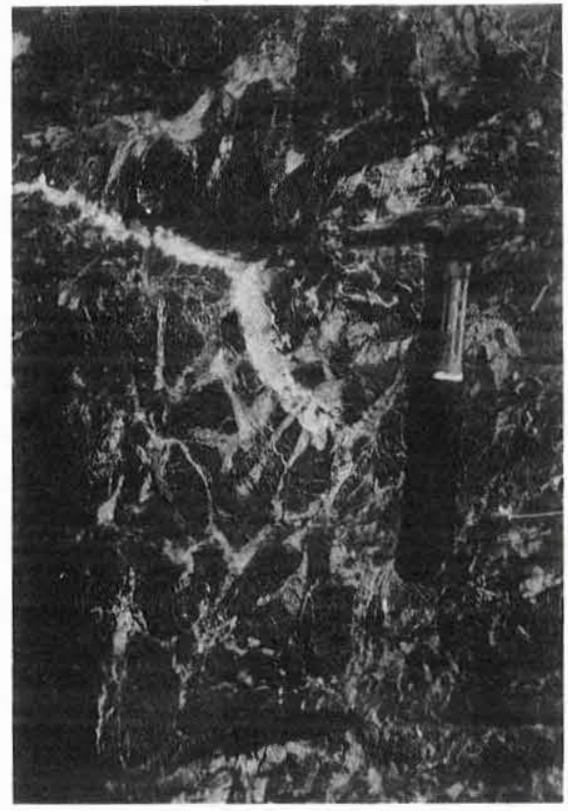

Foto 2 - Parte inferior del afloramiento Sur, donde los estromatolitos se encuentran afectados por venas de carbonatos y deformados. Los individuos mustran aparente ramificacíon activa. La superficie no es totalmente paralela al eje mayor de los estromatolitos

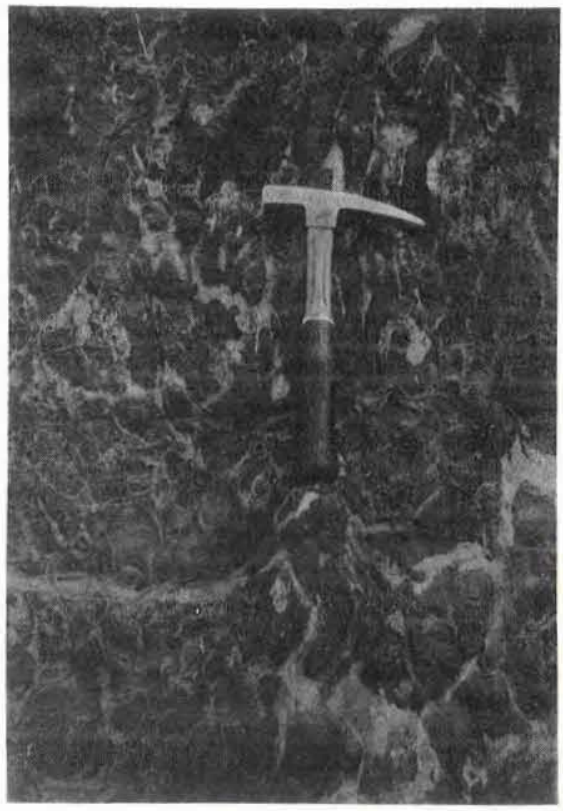

Foto 3 - Idem a Foto 2 pero mostrando formas mas variadas

*The Rock Color Chart, The Geological Society of America, New York, 1963 


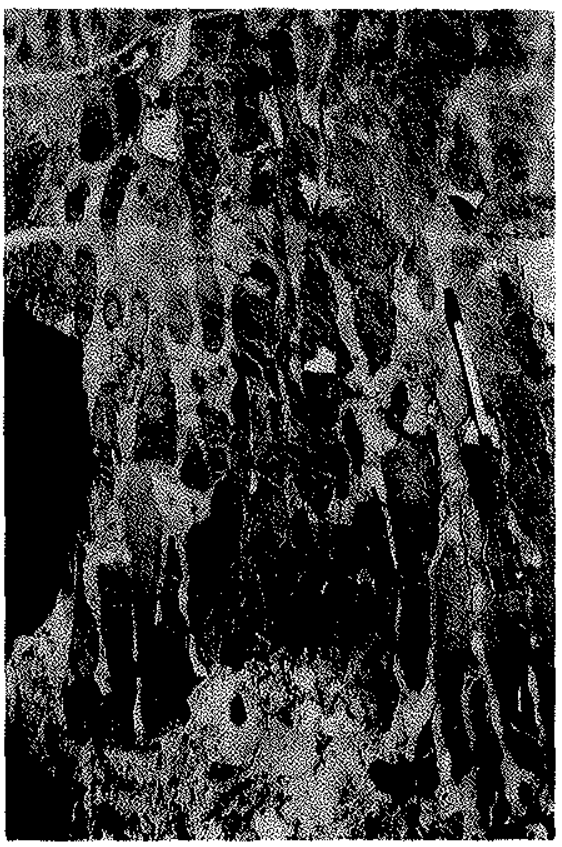

Foto 4 - Parte media-superior del afloramiento Norte. Ya se nota la presencia de formas solitarias y con menos ramificaciones

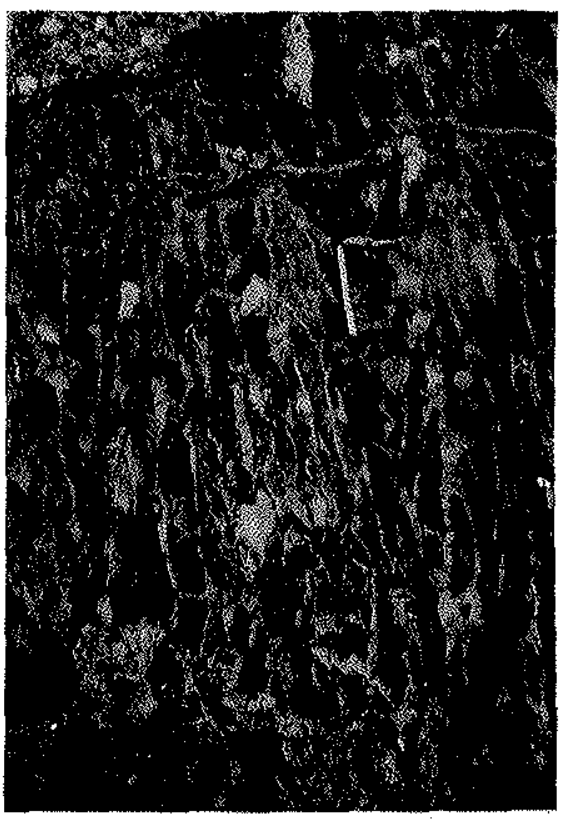

Foto 5 - Idem a Foto 4 pero mostrando la parte superior del afloramiento donde se observan formas solitarias y con escasas ramificaciones

ginoso, lineado y formando "nidos". La laminación (microtextura interna) es debida a la alternancia de cristales mas finos (equigranulares) y mas gruesos, todos esparíticos.

El material que rodea a las estructuras estromatolíticas y que actúan como matriz es subesparita equigranularmhipidiotópica, en zonas varía a inequigranular. El contacto entre las dos zonas (estromatolitos y "matriz") es neto. Los terrígenos (menos del $1 \%$ ) son de tamaño limo fino a arena fina y de composición cuarzosa y plagioclásica. Los cristales de la "matriz", estan orientados aproximadamente paralelas al eje de los estromam tolitos. Posteriormente fueron afectados por venas esparíticas. En partes los cristales estan deformados y han sufrido procesos neomórficos.

Descripción, clasificación, análisis de las estructuras En este estudio usaremos cuatro criterios de clasificación que son los sustentados por Hofman (1969), Logan y otros (1964), Raaben (1969) y Cloud y Semikahtov (1969).

\section{A. DESCRIPCIÓN SIGUIENDO LAS NORMAS DE HOFMAN Láminas:} componentes claros y oscuros.

Microestructura: laminada distintiva (mas del 90\% de los ejemplares).

Tipo de curvatura $y$ orden: liso a ondulado.

Perfil: convexo (prolado a oblado $\mathrm{E}=0,25$ a 4), en un mismo ejemplar puede llegar hasta muy prolado a muy oblado.

Perfil de planta: Redondo (circular, elíptico, ovoide) $67 \%$ de los ejemplares.

Lobado (laxilobado, brevilobado, densilobado) $23 \%$ de los ejemplares.

Poligonal, $6 \%$ de los ejemplares.

Media luna, $3 \%$ de los ejemplares. 
Enlace: varían de parcialmente enlazados a no enlazados.

Espaciado: muy cerca $(\mathrm{P} \leqslant \mathrm{r})$ a cerca $(\mathrm{P} \leqslant 2 \mathrm{r})$.

Relieve (relativo): alto $(2 \mathrm{r} \ll \mathrm{h})$, escasos moderados $(2 \mathrm{r} \approx \mathrm{h})$.

Factor de crecimiento columnar: (turbinados, cilíndricos y terete) $\mathrm{G}=0,8$ a 0,41 .

Variabilidad: alargados, con constricciones, y desiguales; anchos y cortos.

Posición: principalmente rectos y erectos, hay escasos sinuosos.

Estilo de ramificación: no ramificados a ramificados (generalmente pasivos).

Superficie de ornamentación: lisa.

Caracteres internos: pared?

Espesor de lámina interna: generalmente constante, $\mathrm{T}=\mathrm{mm}$.

Espacio de las láminas (2r) ancho del estromatolito: 1 a $10 \mathrm{~cm}$, promedio: 1 a $5 \mathrm{~cm}$.

Alto de los estromatolitos: $20 \mathrm{~cm}$ a $65 \mathrm{~cm}$ (mas raros), a veces es dificil de evaluar porque se desplazan y no se puede seguir sus ramificaciones.

Material: carbonático, calcita.

B. SEGÚN LOGAN, REZAK Ý GINSBURG Tendríamos dos tipos principales de formas geométricas que se combinan y alternan de diferente manera: LLH y SH. En nuestros yacimientos las formas cambian vertical y lateralmente. A continuación describiremos las características esenciales de la colonia y sus variaciones, para eso introduciremos un término propuesto por Kendall y Skipwith (1968) en Hofman (1969), para hemisferoides apilados que crecen invertidos (SH-I), que es la forma inversa de $\mathrm{SH}-\mathrm{V}$. También utilizaremos para indicar las variaciones, no solo las escrituras de los símbolos geométricos en forma lineal, sino su variación vertical.

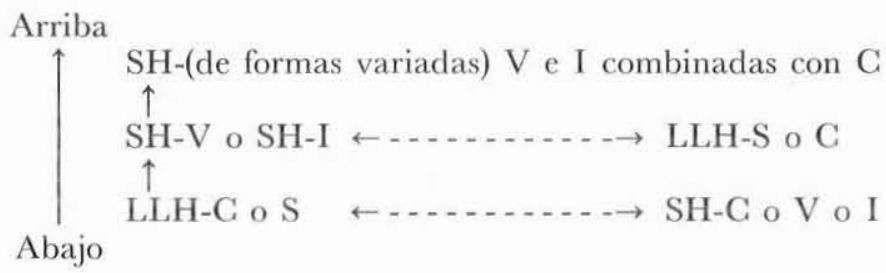

Estas "fórmulas" expresadas anteriormente de ninguna manera muestran todas las variaciones de los cambios laterales verticales que se presentan en los yacimientos sino características generales. Los mismos necesitarían para su mejor expresión y dada su variedad una mayor cantidad de términos geométricos o combinaciones de ellos, lo cual complicaría enormemente la nomenclatura, que no es la intención ni el objetivo de este trabajo.

Hay que hacer notar que la utilización de esta clasificación simplifica un problema que no es tán fácil encerrarlo en términos geométricos exclusivamente y su relación con el medio ambiente. Porque en este caso una gran variedad de formas y combinaciones, que se encuentran dentro de una masa "carbonática" sin mayores variaciones texturales ni composicionales alternan vertical y lateralmente con otras formas y si utilizamos el criterio sustentado por Logan et al. (1964) nos encontramos que tendríamos una gran variedad de microambientes en pequeña escala que son dificil de explicar. Además, pensamos que la característica ambiente intertidial con acción de oleajes dadas por el tipo LL-H varían alternativamente y en pocos centímetros (vertical y lateralmente) a ambiente intertidal, donde la acción del oleaje y otros factores evitan el crecimiento de algas entre los estromatolitos (SH). Por último, aunque se utilice esta clasificación exclusivamente el problema del cronoestratígrafo queda sin solución, y pensamos que ántes de descartar la utilización de los estromatolitos como indicadores y zonadores de edad debemos hacer un esfuerzo. Por lo tanto momentaneamente y hasta que se llege a la solución del problema 
se propone que los estromatolitos se clasifiquen con la fórmula ambiental de Logan et al. (op. cit.) complementando además con otras clasificaciones que den nombres y características de Supergrupo o grupo (género), formas (especies) y utilizando elementos de análisis y comparación descriptivos standarizados. Aún empleando estas clasificaciones no se llega a una solución totalmente inequívoca y satisfactoria.

C. Las conclusiones, siguiendo las clasificaciones de Hofman, Raaben y Cloud y Semikhatov (op. cit.) se encuentran resumidas en el Cuadro I.

Cuadro I

\begin{tabular}{lccccc}
\hline \multicolumn{1}{c}{ Grupo } & Autor & Edad & Autor & Edad & Autor \\
\hline & \multicolumn{1}{c}{ Cloud-Semikhatov } & Raaben & & Hofman \\
Boxonia Koro & $X$ & $950-570$ & $X$ & $675-600$ & $?$ \\
Eucapsiphora nuev. gen. & $?$ & 1600 & s.d. & - & s.d. \\
Gymnosolen Stei. & $X$ & $950-675$ & X & $750-700$ & X \\
Inzeria Krylov & $?$ & $950-675$ & $X$ & $960-900$ & $?$ \\
Jurusania Krylov & $?$ & $950-675$ & s.d. & $960-900$ & $?$ \\
Minjaria Krylov & s.d. & $950-675$ & X & $760-680$ & $?$ \\
Kusiella & s.d. & 1800 & c.s. & $1500-1400$ & $?$ \\
Katavia & s.d. & $950-650$ & A.c.s. & - & $?$ \\
\hline
\end{tabular}

$\mathrm{X}$ : determinado como afin a; ?: dudoso; s.d.; sin descripción; c.s.: pertenece a otro supergrupo; A: edad asignąda en Australia

Ambiente de sedimentación Las carbonatitas del grupo Bambú demuestran que ellas se han depositado en mares someros y cálidos, principalmente en la zona tidal (supratidal y subtidal), con variaciones energéticas cíclicas, protegida de la acción del oleaje, lo cual depende de su posición paleogeográfica. Por la observación de sus estructuras estromatolíticas del yacimiento aquí descripto, siguiendo a Hubbard (1972), podemos concluir que las mismas nos indican un crecimiento algal constante igual o mayor que la acumulación de sedimentos y sin una orientación preferencial de la corriente (comparar con las características dadas por Logan y otros anteriormente). La escasez de elementos terrígenos (tamaño arena) parece indicar intensa meteorización química y/o la ausencia de areas de gran relieve $\mathrm{y} / \mathrm{o}$ que las mismas se encontraban relativamente distantes.

Edad y consideraciones generales Las rocas preCámbricas son consideradas en general no fosiliferas, aunque faunas de metazoas (faunas de Ediacara) rastros y cuerpos (dubiofósiles y fósiles) que se presentan en el Infracámbrico hasta el Eocámbrico (incluyendo Vendiano), son frecuentemente mencionadas. La gran cantidad y distribución de los estromatolitos durante el preCámbrico al Ordovícico Inferior y su declinación durante el Fanerozoico parece responder a una relación inversa. A pesar de que los estromatolitos se presentan en ambientes variados, su registro fósil está areal y ecológicamente restringido, a ambientes subacueos con aereación y condiciones que permitan un desenvolvimiento de los organismos formadores de los mismos. La declinación de estas bioestructuras puede ser explicada en parte por la evolución y diversificación a partir del Fanerozoico de organismos que actúan sobre las algas, se alimentan sobre su superficie y que conjuntamente con animales cavadores destruyen las estructuras orgánico-sedimentarias.

Mucho se ha discutido y se discute sobre la edad de las sedimentitas del Grupo Bambuí, los datos más recientes ubican a las mismas dentro del Cámbrico más bajo hasta el preCámbrico más joven (Cuadro II). 
Cuadro II

\begin{tabular}{|c|c|c|c|c|}
\hline Autor & Localidad & Restos identificados & Edad & $\begin{array}{l}\text { Análisis } \\
\text { ambiental } \\
\text { realizado }\end{array}$ \\
\hline Casedanne (1964) & $\begin{array}{l}\text { Morro de } \\
\text { Chapéu, } \\
\text { oeste de } \\
\text { Jacobina (BA) }\end{array}$ & Collenias & $\begin{array}{l}\text { PreCámbrico } \\
\text { Sup. a Cámbrico } \\
\text { Inf. }\end{array}$ & $\mathrm{Si}$ \\
\hline Casedanne (1965) & Inhaúma (MG) & Solenoporaceas & $\begin{array}{l}\text { PreCámbrico } \\
\text { Sup. a Cámbrico } \\
\text { Inf. }\end{array}$ & $\mathrm{Si}$ \\
\hline Casedanne (1968) & Vazante (MG) & Collenias & - & $\mathrm{Si}$ \\
\hline $\begin{array}{l}\text { Dardenne }(1970) \\
\text { y otros }\end{array}$ & $\begin{array}{l}\text { Vazante- } \\
\text { Paracatu, } \\
\text { Distrito } \\
\text { Federal } \\
\text { (MG-Goiás) }\end{array}$ & $\begin{array}{l}\text { algae mats-LLH } \\
\text { SH-conophyton }\end{array}$ & PreCámbrico Sup. & $\mathrm{Si}$ \\
\hline Dardenne (1972) & Vazante (MG) & $\begin{array}{l}\text { LLH-SH-cilindricos- } \\
\text {-cónicos }\end{array}$ & PreCámbrico Sup. & $\mathrm{Si}$ \\
\hline $\begin{array}{l}\text { Cloud y Dardenne } \\
\text { (1973) }\end{array}$ & $\begin{array}{l}\text { Vazante, } \\
\text { Cabeludo (MG) }\end{array}$ & $\begin{array}{l}\text { Conophyton aff. } \\
\text { C. Metula Kirie- } \\
\text { chenko. }\end{array}$ & $\begin{array}{l}\text { Rifeano Med. } \\
(1350-1950) \\
\text { mas probable } \\
\text { alrededor de } \\
1000\end{array}$ & - \\
\hline Sommer (1969) & $\begin{array}{l}\text { Pedro Leopoldo } \\
\text { (MG) }\end{array}$ & $\begin{array}{l}\text { Microfósiles } \\
\text { algae incertae } \\
\text { (Bambuites } \\
\text { erichseini) }\end{array}$ & - & - \\
\hline Sommer (1971) & $\begin{array}{l}\text { Pedro Leopoldo } \\
\text { (MG) }\end{array}$ & $\begin{array}{l}\text { Bioglifos } \\
\text { (Genkinneiyia } \\
\text { Walcott.) }\end{array}$ & $\begin{array}{l}\text { Algonquiano } \\
\text { Sup. }\end{array}$ & - \\
\hline Este estudio & $\begin{array}{l}\text { Sete Lagoas, } \\
\text { Santa Helena } \\
\text { (MG) }\end{array}$ & $\begin{array}{l}\text { Supergrupo } \\
\text { Gymnosolenida }\end{array}$ & $\begin{array}{l}\text { Rifeano Sup. } \\
(950-650)\end{array}$ & $\mathrm{Si}$ \\
\hline \multicolumn{3}{|c|}{$\begin{array}{l}\text { Estudio Radiométrico de Amaral-Kawashita (1967) en } \\
\text { pelitas de la localidad de Vazante, método Rb-Sr. }\end{array}$} & $600 \pm 50$ & - \\
\hline
\end{tabular}

En nuestro caso llegamos a los siguientes datos, analizando todos los elementos de juicio y criterios sustentados por Raaben, Cloud y Semikhatov y Hofman (1969) y excluyendo a Logan et al. (1964), por no tener implicancia geocronológica.

Los datos tabulados anteriormente nos demuestran que las descripciones surgidas de Hofman se asemejan a Gymnosolen, su asignación a otros grupos es dudosa.

Según la comparación con las descripciones de Cloud y Semikhatov, pertenecerían a los grupos Boxonia y Gymnosolen, sus semejanzas con otros grupos son dudosas, pero no se descartan. Del análisis de lo expresado por la clasificación de Raaben estaríamos ante la presencia del supergrupo Gymnosolenida representados principalmente por Boxonia, 


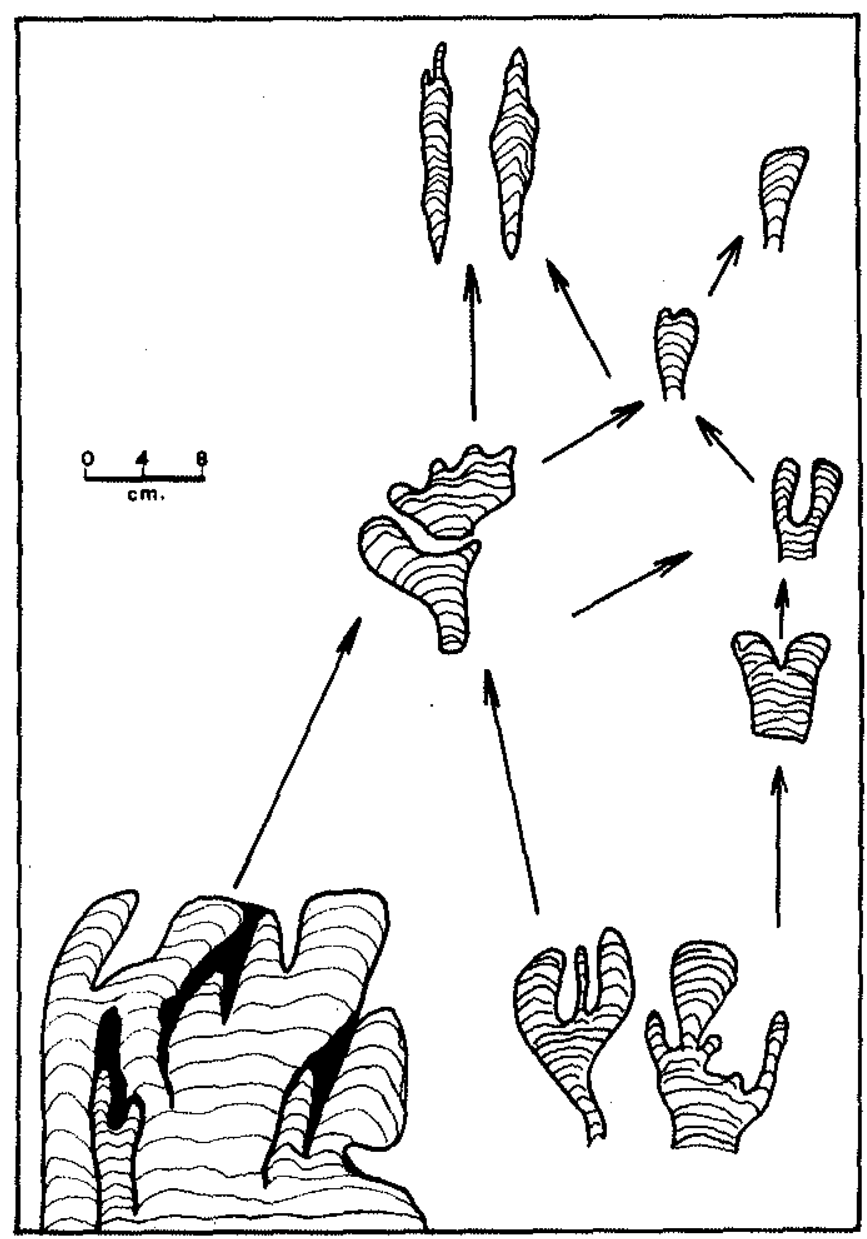

Figura 2 - Bosquejo esquemático mostrando la variácion de formas y ramificaciones, que ocurren en el yacimiento estromatolítico Sur, desde la base hacia el techo

Gymnosolen, Inzeria y Minjaria. Su semejanza en las descripciones con los dos primeros parece más probable

Todos estos datos y las edades asignadas por los diterentes autores nos estan señalando una edad: Rifeano Superior (950 a 650 m.a.)

Esta primera conclusión, por la comparación de los autores anteriormente citados, necesita una revisión más detallada de parte de los especialistas en el estudio de los estromatolitos. Por ejemplo, si tratamos individuos aislados podriamos tener en nuestro caso hacia el techo otro supergrupo? - Conophytonida? o en la parte inferior Tungussida? o Kusiella? etc. Nosotros hemos tratado de caracterizar estadísticamente el yacimiento y de acuerdo a esto clasificarlo, pero no todas las paredes cortan paralelalmente a los estromatolitos y a eso se puede deber parte de la variación de grupos enunciados. Además se ha evitado la creación de nuevos grupos, géneros o especies concientes de que esta posibilidad no debe descartarse en un análisis posterior y teniendo también en cuenta que no todos los estromatolitos necesariamente tienen valor cronoestratigráfico y que sus descripciones no siempre se encuentran en la bibliografia consultada, ni son fáciles de conseguir. Los datos expresados en el Cuadro II y las conclusiones de este estudio señalan sin ninguna duda la edad preCámbrica de las rocas calcáreas del Grupo Bambuí (Formación Sete Lagoas y/o Paraopeba) esto está principalmente confirmado por la presencia de Cono- 
Figura 3 - Idem a Fig. 2, pero referido al afloramiento estromatolítico Norte. En el techo se bosqueja el corte transversal de los estromatolitos

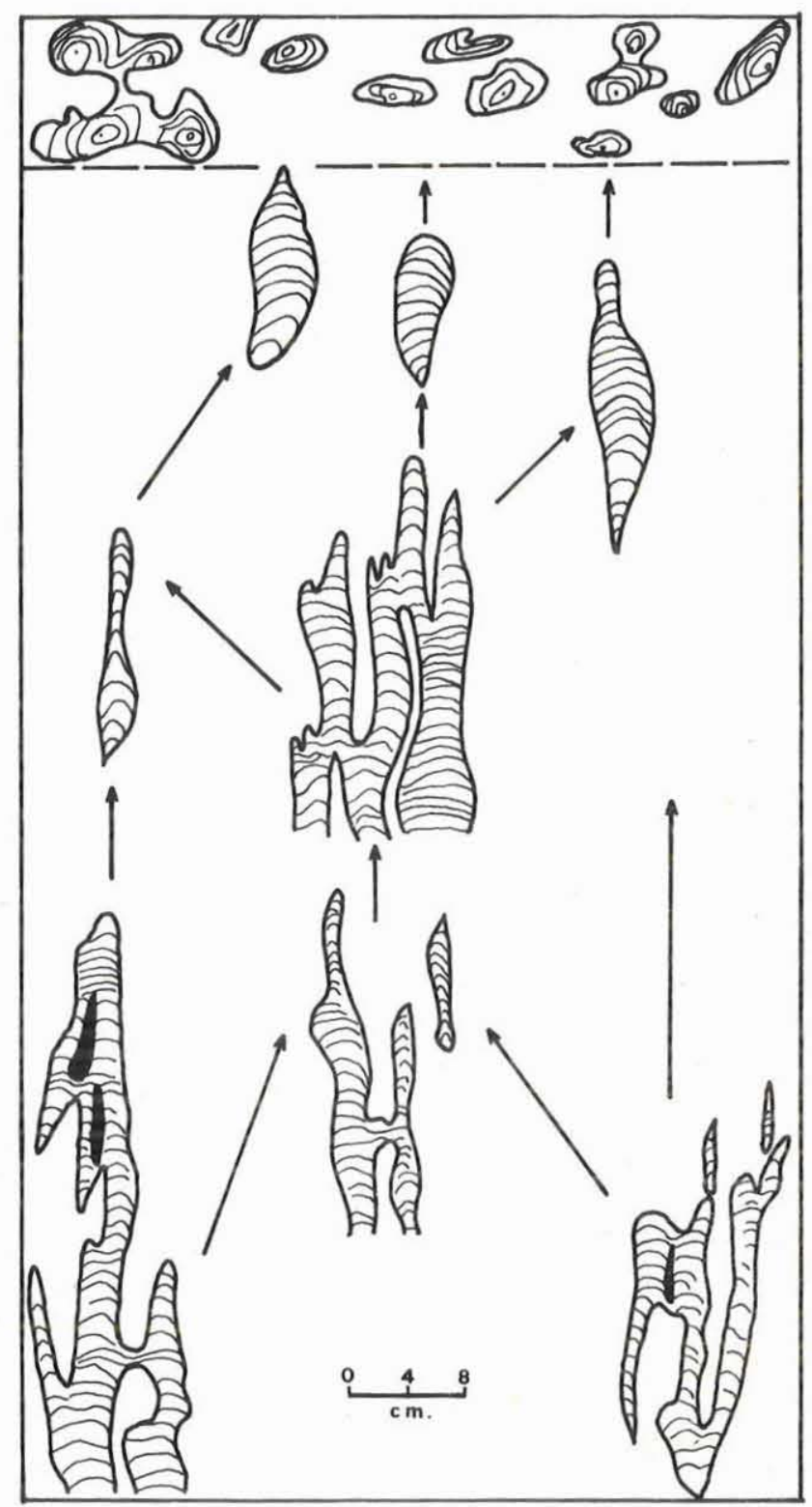

phyton encontrados por Dardene en Cabeludo (Minas Gerais) y Goiás (San Gabriel) y que es un fósil indicativo del preCámbrico, aunque Conophyton basalticum y C. cf. gaubitza llegan al Vendiano superior.

Los datos examinados nos indican que el Grupo Bambuí puede ser ubicado dentro del Rifeano Medio (su parte superior) a Rifeano Superior es decir que su rango oscila hasta los últimos datos examinados en base a los estromatolitos desde 1000 a 650 millones de años. Su rango puede llegar hasta el Vendiano pero no supera la edad de 570 millones de años límite del comienzo Cámbrico.

Además para avalar la edad preCámbrica nos queda el análisis empírico, que consiste en que los datos geoquímicos encontrados hasta ahora en las rocas calcáreas del Grupo 


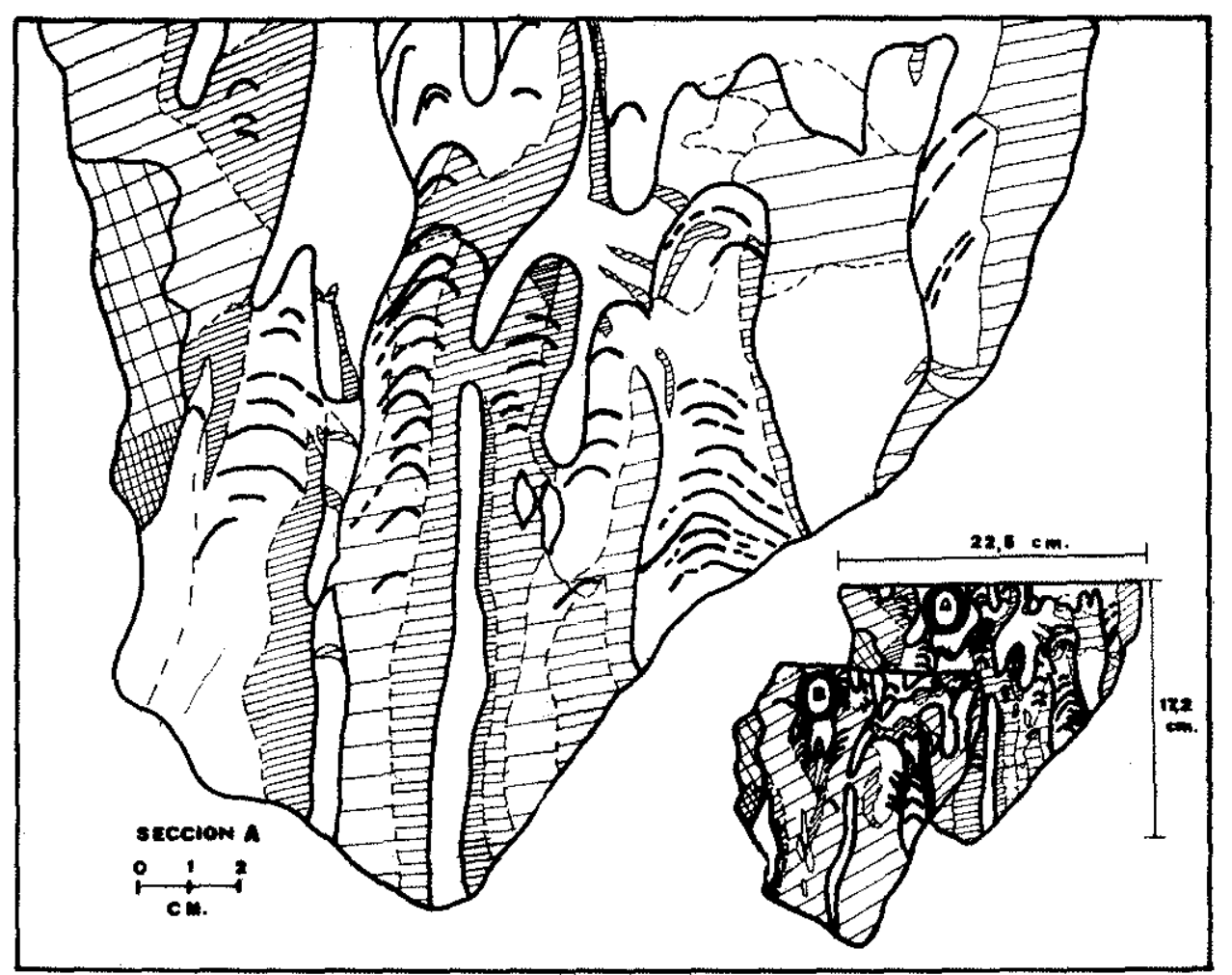

Figura 4 - Seccín o perfil A y diagrama donde se observa la relación entre los perfiles seriados. Superficies cortadas, lisas y atacadas con ácido clorhídrico para obtener mayor diferenciacion entre los estromatolitos y el material que los "rodea". En general, las estructuras están enmascaradas por venas de carbonatos y procesos neomórficos (areas cuadriculadas y rayadas con diferente intensidad). Yacimiento Norte

Bambuí no demostrarían un ambiente que inhibiera otras formas orgánicas con exclusión de los estromatolitos. Es llamativo que en asociaciones con iguales características litológicas del Cámbrico hasta la actualidad, se presenten restos orgánicos convencionales. Es válido pensar que la ausencia de los mismos y la aparente "exclusividad" de la presencia de los estromatolitos indicaría un desarrollo de una asociación biológica incipiente de microorganismos y algas donde la fotosíntesis actuaba en un ambiente sedimentario calcáreo-dolomítico apto. Pero en el cual, las condiciones de evolución no permitieron en ese momento el desarrollo evolutivo y/o la conservación de otros organismos formadores de esqueletos, que "aparecen" bien identificados a partir de Cámbrico (mayor de 570 millones de años).

Resumen de las características principales de los estromatolitos estudiados 1 1) En una misma colonia hay una migración de características e incluso en un mismo ejemplar. La parte mas baja del yacimiento comienza con partes mas gruesas y ramificadas activamente, mientras que hacia el techo se ramifican pasivamente hasta presentarse sin ramificación y más delgados. En un mismo ejemplar se puede observar que la textura interna (laminación) varía desde cónica apical muy angulosa (tipo conophyton) hasta cóncava achatada. 


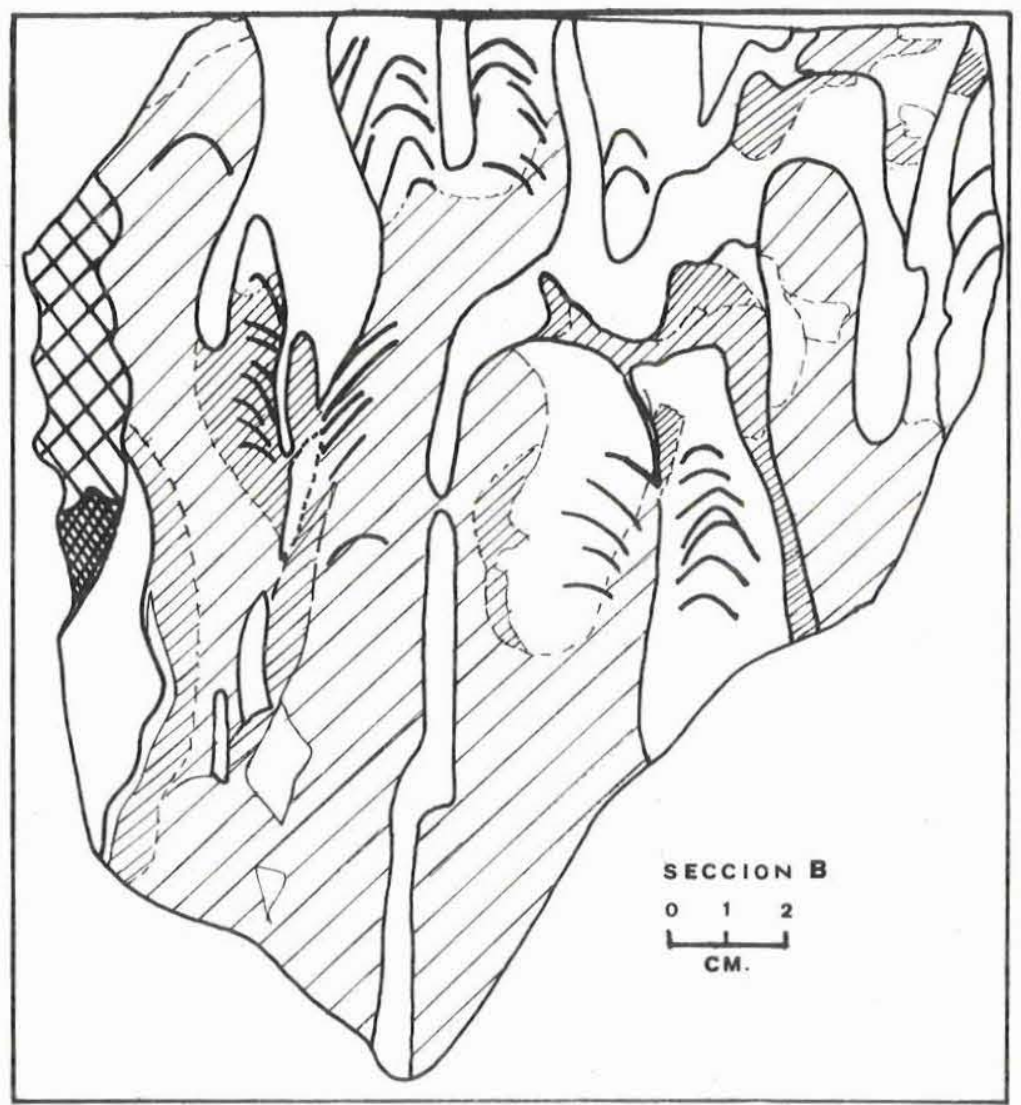

Figura 5 - Sección o perfil seriado B. Idem Fig. 4, ver su ordenamiento en el diagrama al pie de dicha figura

2) Muchos ejemplares se conectan lateralmente (coalescen) para formar una estructura alargada donde luego se desarrolla una sola rama o casi sin ramificación.

3) Los ejemplares son alargados y con contricciones. A veces se observa "pared".

4) Crecen paralelalmente pero se desplazan lateralmente en su crecimiento.

5) Ausencia total de estructuras en las rocas encajantes a pesar del gran desarrollo y lo bien marcadas láminas internas de los estromatolitos.

CONSIDERACIONES GENERALES De los datos presentados anteriormente podemos extraer algunas conclusiones e hipótesis de trabajo para estudios posteriores.

La migración o variaciones en las características de un mismo yacimiento puede ser debido a varios factores o circunstancias que actúen conjuntas y/o parcialmente. Hay que tener en cuenta que éstas modificaciones señaladas dificultan las tareas de clasificación y descripción y puede conducir a conclusiones erróneas. Por ejemplo, los resultados, del estudio de un yacimiento donde solamente se presente la parte superior (o media o inferior) de una asociación estromatolítica como la descripta, arribarán a conclusiones falsas.

Algunas de las posibilidades para su explicación pueden ser:

a) Durante el desarrollo del estromatolito los individuos mas seniles migran hacia formas más jóvenes, b) a la coexistencia de varios grupos de organismos (algas-microfósiles), 
c) pequeñas variaciones ambientales no detectadas macroscópicamente (sin cambio de composición ni variación en las estructuras sedimentarias), que inhiben algunas formas favoreciendo el desarrollo de otras. Todas estas incógnitas y sus combinaciones son importantes de ser analizadas y tenidas en cuenta en futuros trabajos por los investigadores del Grupo Bambuí y de otras formaciones similares en otros lugares del país y donde se presenten estromatolitos.

La comparación de las diferentes asociaciones de estromatolitos descriptas en formaciones calcáreas' preCámbricas (Bambuí, São Roque, Açungui, Corumbá) merecen ser tenidas en cuenta por los paleontólogos y estratígrafos. Ellas deben ser estudiadas y comparadas con un criterio de descripción y clasificación similar, uniforme y amplio. Los resultados de estos estudios seguramente esclarecerán la correlación entre ellas y por consiguiente su estratigrafia y su posterior intento de correlación con otras formaciones con similares incógnitas y con asociaciones estromatolíticas (Grupo Tandil, Argentina). A esta revisión debe sumarse los esfuerzos en la continuación de los estudios de los microfósiles, su búsqueda sistemática y su clasificación. Los mismos son de gran importancia en la solución de problemas del preCámbrico donde la escasez de restos orgánicos convencionales hacen dificil develar su cronoestratigrafía.

Por último debe quedar claramente expresado en este trabajo, solamente trata de ser una contribución sobre un tema de gran interés e importancia, pero que el mismo no es exhaustivo ni completo, sino que intenta aportar elementos de juicio e hipótesis para futuros trabajos. Además debe tenerse en cuenta que la utilización de los estromatolitos como indicadores estratigráficos es más que una discusión teórica, un problema empírico.

Agradecimientos El autor esta muy agradecido al Conselho Nacional de Pesquisas, de Brasil, bajo cuyo auspicio y sulcención realizó este trabajo, asimismo hace extensivos sus agradecimientos al Consejo de Investigaciones de la Kepública Argentina, que por medio del convenio existente entre ambos permitió su participación en dicho estudio.

Debo especial reconocimiento al Dr. Roberto Cardoso que me acompañó en los trabajos de campo en Minas Gerais, que gentilmente me allanó todas las dificultades.

Asi mismo debo mis agradecimientos a los colegas Dres. Dardenne Farias y Ferreira de Andrade por acompañarme durante los trabajos de campo en Goiás y Distrito Federal y mostrarme interesantes yacimientos de estromatolitos descubiertos por ellos y actualmente en estudio por los citados.

Por último mi cordial agradecimiento al Prof. Fernando Flávio Marques de Almeida por la lectura crítica y objetiva del manuscrito y por su apoyo moral y técnico durante mi trabajo.

\section{REFERENCIAS}

AMARAL, G, y KAWASHITA, K. - 1967 - Determinação da idade do Grupo Bambuí pelo método Rb-Sr, Anais do XXI Cong. Bras. de Geol., Curitiba

BRANCO, J, RODRIGUEZ. y COSTA, T. - 1961 - Roteiro para a Excursão Belo Horizonte-Brasilia, Publicación N. ${ }^{\circ} 15$ Contribución al XIV Cong. Bras. de Geología, Escola de Enge. nharia Universidad de MG. Instituto de Pesquisas Radiativas

CASEDANNE, J. - 1964 - Biostrome a Collenia dans le calcaire de Bambui. Anais Acad. Bras. Giên. 36 (1): 49-58, Río de Janeiro

CASEDANNE, J. - 1965 - Découverte d'algue dans le calcaire de Bambuí, Etat de Minas Gerais, Brasil. Anais Acad. Bras. Ciên., 37 (1): 79-81

CASEDANNE, J. - 1968 - Description du Biostrome et Collenia de la mine de Vazante (M.G.), Anais Acad. Bras. Cien., 40(2): 215-225, Río de Janeiro

CLOUD Jr., P. E. y SEMIKHATOV, M. A. - 1969 - Proterozoic Stromatolite Zonation Amer. Jour. Sci., 267: 1017-1061 
CLOUD, P. y DARDENNE, M. - 1973 - Proterozoic Age of the Bambui Group in Brasil, Geol. Soc. of Amer., Bull., 84: 1673-1 676

CHENG, YEH. CHI., CHI-CHIANG, HO. y KUO-CHANG, SUN - 1969 - Sedimentation of Sinian algal carbonate rocks. Traducción de Acta Geol. Sínica, 45(4): 416-431 en Internat. Geol. Rev., 1969, 11(5)

DARDENNE, M. - 1972 - Os recifes Algais da Região de Vazante (M. G.), Brasil, Belém 1972. Anais XXVI Cong. Bras. Geol.

DARDENNE, M. A., MELO, M. G. y MOERI, E. - 1970 - Os estromatolitos do Grupo Bambuí Importância Estratigráfica e Metalogênica. Resumen del XXV Cong. Bras. Geol., San Pablo

HOFMAN, H. J. - 1969 - Attributes of Stromatolites, Geol. Survey of Canada, Dep. of Eng. Min. and Resources. Paper 69-39

HUBBARD, J. A. E. B. - 1972 - Stromatolic Fabric: A Petrogtaphic Model, $24^{\text {th }}$. Intern. Geol. Congress, Section 7

LOGAN, B. W., REZAK, R. y GINSBURG, R. N. - 1964 - Classification and enviromental significance of algal stromatolites, Jour. Geol., 72(1): 68-83

MARCHESE, H. G. - 1974 - Litoestratigrafia y petrología del Grupo Bambuí en los Estados de Minas Gerais y Goiás, Brasil. Rev. Bras. Geoc., 4(3): 172-190, São Paulo

RAABEN, M. E. - 1969 - Columnar stromatolites and late Precambrian Stratigraphy, Amer. Jour. Sci., 267: 1-18

SOMMER, F. W. - 1969 - Bióglifos do Calcário Bambuí de Pedro Leopoldo, M. G. Anais Acad. Bras. Ciên. 41(4), Rio de Janeiro

SOMMER, F. W. - 1971 - Microfósseis do Calcário Bambuí de Pedro Leopoldo M.G. Anais Acad. Bras. Cien. 43

WALTER, M. R. y PREISS, W. V. - 1972 - Distribution of stromatolites in the Precambrian and Cambrian of Australia, $24^{\text {th }}$, Int. Geol. Congress, Section 1 